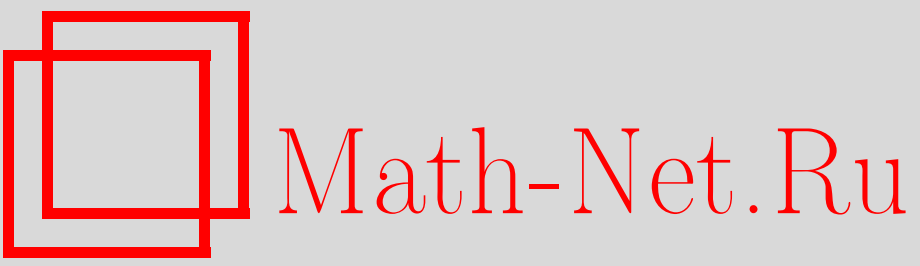

В. Н. Туняк, Релятивистская теория гравитации и скалярное поле БрансаДикке, ТМФ, 1996, том 106, номер 2, 325-332

DOI: https://doi.org/10.4213/tmf1119

Использование Общероссийского математического портала Math-Net.Ru подразумевает, что вы прочитали и согласны с пользовательским соглашением

http://www . mathnet.ru/rus/agreement

Параметры загрузки:

IP: 34.227 .88 .159

26 апреля 2023 г., 06:59:09 
ТЕОРЕТИЧЕСКАЯ

И МАТЕМАТИЧЕСКАЯ

ФИЗИКА

Том 106, № 2

февраль, 1996

\section{В.Н. Туняк}

\section{РЕЛЯТИВИСТСКАЯ ТЕОРИЯ ГРАВИТАЦИИ И СКАЛЯРНОЕ ПОЛЕ БРАНСА-ДИККЕ}

Рассматривается естественное обобщение релятивистской теории гравитации (РТГ) путем включения в нее скалярного поля Бранса-Дикке. Уравнение скалярно-тензорного гравитационного поля в пространстве Минковского и выражение суммарного метрического тензора энергии-импульса гравитационного поля и негравитационной материи выводятся из вариационного принципа с гравитационным лагранжианом, квадратичным по первым производным скалярного и тензорного гравитационных потенциалов. Получено двухпараметрическое сферически-симметричное статическое решение вакуумных уравнений с нулевой массой тензорного гравитона. Это решение имеет истинно сингулярную поверхность Шварцшильда. В случае ненулевой гравитонной массы получено приближенное несингулярное решение для начала Вселенной. Отмечено, что в рамках скалярно-тензорного обобшения РТГ несингулярная однородная изотропная космология может быть представлена не только циклическими моделями, но и моделями с неограниченным расширением Вселенной и одновременным уменьшением гравитационного скаляра.

Целью данной работы является рассмотрение естественного обобщения релятивистской теории гравитации (РТГ) [1-3], обеспечивающего включение в РТГ скалярного гравитационного поля Бранса-Дикке [4]. В соответствии с общей теоретической концепией РТГ принимаются постулаты о сушествовании физического пространства-времени Минковского $E_{4}$ и об эффективном характере риманова пространства $V_{4}$. Скалярно-тензорное гравитационное поле в пространстве $E_{4}$ описывается как скалярным потенциалом $\psi$, так и тензорными потенциалами

$$
\Phi^{\mu \nu}=\psi \sqrt{g / \gamma} g^{\mu \nu}-\gamma^{\mu \nu}
$$

При этом $g_{\mu \nu}$ - метрический тензор $V_{4}, g=\operatorname{det}\left(g_{\mu \nu}\right), \gamma_{\alpha \beta}$ - метрический тензор $E_{4}$, $\gamma=\operatorname{det}\left(\gamma_{\alpha \beta}\right)$. Потенциалы $\Phi^{\mu \nu}$ подчиняются дополнительным квазигармоническим условиям

$$
\nabla_{\mu} \Phi^{\mu \nu}=0
$$

$\nabla_{\mu}$ обозначает ковариантную производную со связностью Кристоффеля в пространстве $E_{4}$.

Исходим из вариационного принципа

$$
\delta J=(1 / c) \delta \int L \sqrt{-\gamma} d^{4} x=0
$$


где

$$
\begin{aligned}
L & =L_{g}+\sqrt{g / \gamma} L_{m}=L_{\psi R}+L_{\mu}+\sqrt{g / \gamma} L_{m}, \\
L_{\psi R} & =-\left(k_{\omega}^{2} / 2 \varkappa\right)\left\{\psi R-(\omega / \psi) \partial_{\alpha} \psi g^{\alpha \beta} \partial_{\beta} \psi\right\}, \\
L_{\mu} & =\left(\mu^{2} k_{\omega}^{2} / 2 \varkappa\right)\left(1+\psi^{2} \sqrt{g / \gamma}-(1 / 2) \psi \sqrt{g / \gamma} g^{\alpha \beta} \gamma_{\alpha \beta}\right),
\end{aligned}
$$

$L_{m}$ - лагранжева функция негравитационной материи в пространстве $V_{4}, R$ - скалярная кривизна $V_{4}, \omega$ - положительная [5] безразмерная константа скалярно-тензорной теории, $k_{\omega}^{2}=(2 \omega+4)(2 \omega+3)^{-1}, \quad \varkappa-$ эйнштейновская гравитационная константа, $\mu$ - константа, которая характеризует массу тензорного гравитона. Известные эффекты отклонения лучей света и запаздывания сигналов в гравитационном поле Солнца показывают, что $\omega>500$ [6].При некоординатных инфинитезимальных калибровочных преобразованиях

$$
\left.\delta_{\varepsilon} \psi=-\varepsilon^{\sigma} \partial_{\sigma} \psi, \quad \delta_{\varepsilon} \Phi^{\mu \nu}=2 f^{\sigma(\mu} \nabla_{\sigma} \varepsilon^{\nu}\right)-\nabla_{\sigma}\left(f^{\mu \nu} \varepsilon^{\sigma}\right)
$$

где

$$
f^{\mu \nu}=\gamma^{\mu \nu}+\Phi^{\mu \nu}, \quad f_{\rho \lambda} f^{\lambda \sigma}=\delta_{\rho}^{\sigma},
$$

$L_{g}(3)$ изменяются по закону

$$
L_{g} \rightarrow L_{g}+\nabla_{\sigma}\left\{\varepsilon^{\sigma}\left[\frac{\mu^{2} k_{\omega}^{2}}{2 \varkappa}-L_{g}\right]-\frac{\mu^{2} k_{\omega}^{2}}{2 \varkappa} \psi_{\lambda \nu} f^{\lambda \sigma} \varepsilon^{\nu}\right\}
$$

После элиминации из $L_{g}(3)$ дивергенциального члена

$$
L_{d}=\left(k_{\omega}^{2} / 2 \varkappa\right) \nabla_{\alpha}\left\{\psi \sqrt{g / \gamma}\left[g^{\alpha \beta} \Pi_{\beta \rho}^{\rho}-g^{\beta \sigma} \Pi_{\beta \sigma}^{\alpha}\right]\right\},
$$

где $\Pi_{\mu \nu}^{\alpha}$ - разность связностей Кристоффеля в $V_{4}$ и $E_{4}$, получаем гравитационную функцию Лагранжа, квадратичную по первым производным потенциалов $\psi, \Phi^{\mu \nu}$,

$$
\begin{aligned}
L_{g}= & L_{g}\left(\psi, \Phi^{\mu \nu}, \gamma_{\alpha \beta}, \nabla_{\alpha} \Phi^{\lambda \sigma}, \partial_{\rho} \psi\right)=L_{k v}+L_{\mu} \\
L_{k v}= & -\left(k_{\omega}^{2} / 4 \varkappa\right)\left\{f_{\mu \rho} \nabla_{\alpha} \Phi^{\mu \lambda} \nabla_{\lambda} \Phi^{\alpha \rho}+(1 / 4) f_{\sigma \nu} f_{\lambda \mu} f^{\alpha \rho} \nabla_{\alpha} \Phi^{\mu \lambda} \nabla_{\rho} \Phi^{\sigma \nu}-\right. \\
& \left.-(1 / 2) f_{\mu \sigma} f_{\varepsilon \lambda} f^{\alpha \rho} \nabla_{\alpha} \Phi^{\mu \lambda} \nabla_{\rho} \Phi^{\varepsilon \sigma}\right\}+\left[(\omega+2) / 2 \varkappa \psi^{2}\right] f^{\alpha \beta} \partial_{\alpha} \psi \partial_{\beta} \psi
\end{aligned}
$$

Подчеркнем, что при этом в соответствии с принципом геометризации [1-3] риманова метрика $g_{\mu \nu}$ в функции Лагранжа $L$ рассматривается как эффективная переменная, которая является локальной функцией потенциалов $\psi, \Phi^{\mu \nu}$ и плоской метрики $\gamma_{\alpha \beta}$. Конкретно в соответствии с (1) это означает, что

$$
\begin{aligned}
\delta g_{\alpha \beta}= & \frac{\sqrt{\gamma / g}}{\psi}\left\{\frac{1}{2} g_{\mu \nu} g_{\alpha \beta}-g_{\alpha \mu} g_{\beta \nu}\right\} \delta \Phi^{\mu \nu}-\frac{1}{\psi} g_{\alpha \beta} \delta \psi+ \\
& +\frac{\sqrt{\gamma / g}}{\psi} \gamma^{\mu \rho} \gamma^{\nu \sigma}\left\{g_{\alpha \rho} g_{\sigma \beta}-\frac{1}{2} g_{\alpha \beta} g_{\rho \sigma}+\frac{\psi}{2} \sqrt{g / \gamma} \gamma_{\rho \sigma} g_{\alpha \beta}\right\} \delta \gamma_{\mu \nu}
\end{aligned}
$$


В результате варьирования по $\psi, \Phi^{\mu \nu}$ получаем уравнения поля

$$
\begin{gathered}
g^{a \beta} \psi_{; \alpha ; \beta}=[\varkappa /(2 \omega+4)] T ; \\
\psi G_{\mu \nu}+g_{\mu \nu} g^{\alpha \beta} \psi_{; \alpha ; \beta}-\psi_{; \mu ; \nu}-(\omega / \psi)\left\{\partial_{\mu} \psi \partial_{\nu} \psi-\right. \\
\left.-(1 / 2) g_{\mu \nu} \partial_{\alpha} \psi g^{\alpha \beta} \partial_{\beta} \psi\right\}+\left(\mu^{2} / 2\right)\left\{\psi^{2} g_{\mu \nu}+\psi \gamma_{\mu \nu}-\right. \\
\left.-(1 / 2) \psi g_{\mu \nu} g^{\alpha \beta} \gamma_{\alpha \beta}\right\}=\left(\varkappa / k_{\omega}^{2}\right) T_{\mu \nu},
\end{gathered}
$$

где точка с запятой обозначает ковариантную производную в $V_{4}, T_{\mu \nu}$ - метрический тензор энергии-импульса негравитационной материи в $V_{4}, T=g^{\alpha \beta} T_{\alpha \beta}, G_{\mu \nu}$ - тензор Гильберта-Эйнштейна. Дифференцируя (6) и учитывая закон сохранения

$$
T_{; \nu}^{\mu \nu}=0,
$$

получаем при $\mu \neq 0$ квазигармонические условия (2). В приближении слабого поля уравнения (5), (6) принимают вид

$$
\begin{aligned}
\square \psi & =[\varkappa /(2 \omega+4)] T, \\
\square \Phi^{\mu \nu}+\mu^{2} \Phi^{\mu \nu} & =\left(2 \varkappa / k_{\omega}^{2}\right) T^{\mu \nu}, \quad \nabla_{\mu} \Phi^{\mu \nu}=0,
\end{aligned}
$$

где $\square=\gamma^{\alpha \beta} \nabla_{\alpha} \nabla_{\beta}$ - оператор Даламбера в $E_{4}$. Таким образом, данная теория описывает скалярно-тензорное поле в пространстве Минковского, которое является смесью (массивного при $\mu \neq 0$ ) тензорного поля $\Phi^{\mu \nu}$ и безмассового скалярного поля $\psi$. В предельном случае $\psi=1, \omega=\infty$ уравнения (5), (6) переходят в уравнения РТГ [1-3].

В соответствии с (4) метрический тензор энергии-импульса негравитационной материи в пространстве $E_{4}$

$$
\Theta_{(m)}^{\mu \nu}=-\frac{2}{\sqrt{-\gamma}} \frac{\delta\left(\sqrt{-g} L_{m}\right)}{\delta \gamma_{\mu \nu}}
$$

имеет вид

$$
\Theta_{(m)}^{\mu \nu}=\sqrt{g / \gamma} T^{\alpha \beta} \frac{\partial g_{\alpha \beta}}{\partial \gamma_{\mu \nu}} .
$$

При использовании уравнений $\Phi$-поля

$$
\frac{\partial L_{g}}{\partial \Phi^{\mu \nu}}-\nabla_{\sigma} \frac{\partial L_{g}}{\partial\left(\nabla_{\sigma} \Phi^{\mu \nu}\right)}=\frac{\sqrt{g / \gamma}}{2} T^{\alpha \beta} \frac{\partial g_{\alpha \beta}}{\partial \Phi^{\mu \nu}}
$$

и соотношения

$$
\frac{\partial L_{k v}}{\partial \gamma_{\mu \nu}}=-\gamma^{\mu \alpha} \gamma^{\nu \beta} \frac{\partial L_{k v}}{\partial \Phi^{\alpha \beta}}
$$

тензор энергии-импульса гравитационного поля

$$
\Theta_{(g)}^{\mu \nu}=-\frac{2}{\sqrt{-\gamma}} \frac{\delta\left(\sqrt{-\gamma} L_{g}\right)}{\delta \gamma_{\mu \nu}}
$$


можно записать в виде

$$
\begin{aligned}
\Theta_{(g)}^{\mu \nu}= & \left(k_{\omega}^{2} / 2 \varkappa\right)\left(\square \Phi^{\mu \nu}+\mu^{2} \Phi^{\mu \nu}\right)- \\
& -\sqrt{g / \gamma} \frac{\partial g_{\alpha \beta}}{\partial \gamma_{\mu \nu}} \frac{k_{\omega}^{2}}{\varkappa}\left\{\psi G^{\alpha \beta}+\left(g^{\alpha \beta} g^{\rho \sigma}-g^{\alpha \rho} g^{\beta \sigma}\right) \psi_{; \rho ; \sigma}-\right. \\
& -(\omega / \psi)\left(g^{\alpha \beta} g^{\rho \sigma} \partial_{\rho} \psi \partial_{\sigma} \psi-(1 / 2) g^{\alpha \beta} \partial_{\sigma} \psi g^{\rho \sigma} \partial_{\rho} \psi\right)+ \\
& +\left(\mu^{2} / 2\right)\left(\psi^{2} g^{\alpha \beta}+\psi g^{\alpha \beta} g^{\beta \sigma} \gamma_{\rho \sigma}-(1 / 2) \psi g^{\alpha \beta} g^{\rho \sigma} \gamma_{\rho \sigma}\right\}
\end{aligned}
$$

При складывании (8), (9) и учете (6) получаем, что суммарный метрический тензор энергии-импульса гравитационного поля и негравитационной материи

$$
\Theta^{\mu \nu}=-\frac{2}{\sqrt{-\gamma}} \frac{\delta(\sqrt{-\gamma} L)}{\delta \gamma_{\mu \nu}}=\Theta_{(m)}^{\mu \nu}+\Theta_{(g)}^{\mu \nu}
$$

имеет вид

$$
\Theta^{\mu \nu}=\frac{k_{\omega}^{2}}{2 \varkappa}\left(\square \Phi^{\mu \nu}+\mu^{2} \Phi^{\mu \nu}\right)
$$

Выражение (10) - слабое соотношение, и поэтому полевые уравнения (6) можно переписать в компактном виде

$$
\square \Phi^{\mu \nu}+\mu^{2} \Phi^{\mu \nu}=\frac{2 \varkappa}{k_{\omega}^{2}} \Theta^{\mu \nu} .
$$

При этом закон сохранения

$$
\nabla_{\mu} \Theta^{\mu \nu}=0
$$

следует непосредственно из квазигармонических условий [2].

В работе [4] сфферически-симметричное статическое вакуумное, $T_{\mu \nu}=0$, решение уравнений (5), (6) с $\mu=0$ было получено при использовании квазидекартовой координатной системы

$$
\left\{\bar{x}^{\alpha}\right\}=\left\{\bar{x}^{0}=c t, \bar{x}^{k}\right\}, \quad \xi^{2}=\bar{x}^{m} \bar{x}^{n} \delta_{m n},
$$

а также римановой метрики и скалярного поля

$$
\bar{g}_{\lambda 0}=e^{2 \alpha(\xi)} \delta_{\lambda 0}, \quad \bar{g}_{k m}=-\delta_{k m} e^{2 \beta(\xi)}, \quad \psi=\psi(\xi)
$$

Решение имеет вид

$$
\begin{array}{cl}
e^{\alpha}=f_{-}^{k_{\omega}} f_{+}^{-k_{\omega}}, & e^{\beta}=f_{+}^{2} e^{n \alpha}, \quad \psi=e^{-\alpha /(\omega+2)} \\
f_{ \pm}=1 \pm\left(r_{0} / \xi k_{\omega}\right), & n=\left[\omega+2-(\omega+1) k_{\omega}\right] /(\omega+2) k_{\omega}
\end{array}
$$

где $M$ - масса системы, $r_{0}=k M / 2 c^{2}, k=\varkappa c^{4} / 8 \pi$. Оно, однако, не удовлетворяет квазигармоническим координатным условиям

$$
\partial_{\alpha}\left(\psi \sqrt{-g} g^{\alpha \sigma}\right)=0
$$

Чтобы найти решение, которое согласуется с этими условиями, введем новые координаты

$$
\left\{\hat{x}^{\alpha}\right\}=\left\{\hat{x}^{0}=c t, \hat{x}^{k}=\left(1+\frac{r_{0}^{2}}{\xi^{2} k_{\omega}^{2}}\right) \bar{x}^{k}\right\}, \quad \zeta^{2}=\hat{x}^{m} \hat{x}^{n} \delta_{m n} .
$$


Таким образом, скалярное поле и риманова метрика принимают вид

$$
\begin{aligned}
\psi & =\psi(\zeta)=F_{+}^{m / 2} F_{-}^{-m / 2}, \\
\hat{g}_{\lambda 0} & =e^{2 \alpha} \delta_{\lambda 0}, \quad \hat{g}_{k m}=n_{k} n_{m}\left(e^{2 \lambda}-e^{2 \nu}\right)-\delta_{k m} e^{2 \lambda}, \\
e^{2 \alpha(\zeta)} & =F_{+}^{-k_{\omega}} F_{-}^{k_{\omega}}, \quad e^{2 \nu(\zeta)}=F_{+}^{m(\omega+1)} F_{-}^{-m(\omega+1)}, \\
e^{2 \lambda(\zeta)} & =F_{+}^{1+m(\omega+1)} F_{-}^{1-m(\omega+1)},
\end{aligned}
$$

где

$$
n_{m}=\hat{x}^{m} / \zeta, \quad F_{ \pm}=1 \pm\left(r_{1} / \zeta\right), \quad m=k_{\omega} /(\omega+2), \quad r_{1}=2 r_{0} / k_{\omega} .
$$

Это решение подчиняется условиям (11), но не является наиболее общим решением такого типа, потому что для него выполняется еще одно дополнительное условие

$$
\alpha+\nu+\ln \psi=0
$$

Для получения более обшего решения, удовлетворяюшего условиям (11), осушествим еше одно координатное преобразование

$$
\left\{x^{\alpha}\right\}=\left\{x^{0}=c t, x^{k}=Y \hat{x}^{k}\right\}, \quad r^{2}=x^{m} x^{n} \delta_{m n},
$$

где $Y$ - произвольная функция $\zeta$. Тогда скалярное поле и риманова метрика принимают вид

$$
\begin{aligned}
\psi & =\psi(r)=F_{+}^{m / 2} F_{-}^{-m / 2}, \\
g_{\lambda 0} & =e^{2 \alpha} \delta_{\lambda 0}, \quad g_{k m}=e_{k} e_{m}\left(e^{2 \sigma}-e^{2 \beta}\right)-\delta_{m k} e^{2 \sigma}, \quad e_{k}=x^{k} / r, \\
e^{2 \alpha(r)} & =F_{+}^{-k_{\omega}} F_{-}^{k_{\omega}}, \quad e^{2 \beta(r)}=(d \zeta / d r)^{2} F_{+}^{m(\omega+1)} F_{-}^{-m(\omega+1)}, \\
e^{2 \sigma(r)} & =(\zeta / r)^{2} F_{+}^{1+m(\omega+1)} F_{-}^{1-m(\omega+1)} .
\end{aligned}
$$

Решение (12) содержит произвольную функцию $\zeta=\zeta(r)$, для определения которой используем условия (11). Последние сводятся к одному уравнению

$$
\frac{\psi^{\prime}}{\psi}+\alpha^{\prime}+2 \sigma^{\prime}-\beta^{\prime}+\frac{2}{r}\left(1-e^{2 \beta-2 \sigma}\right)=0,
$$

штрих означает производную по $r$. Подставляя (12) в (13), получаем следующее уравнение для функции $r=r(\zeta)$ (кстати, очень похожее на соответствуюшее уравнение РТГ $[1$, c.102]:

$$
\frac{d}{d \zeta}\left\{\left(\zeta-r_{1}\right)\left(\zeta+r_{1}\right) \frac{d r}{d \zeta}\right\}=2 r
$$

Его обшее решение имеет вид

$$
r=r(\zeta)=C_{1}\left\{1+\frac{\zeta}{2 r_{1}} \ln \frac{\zeta-r_{1}}{\zeta+r_{1}}\right\}+\frac{C_{2}}{r_{1}} \zeta,
$$


где $C_{1}, C_{2}$ - произвольные постоянные. Формулы $(12),(14)$ - это решение уравнений $(5),(6)$ и (11). Однако координатные условия (11) формально совпадают с квазигармоническими условиями (2) при простейшей метрике $E_{4}$

$$
d \sigma^{2}=\gamma_{\alpha \beta} d x^{\alpha} d x^{\beta}=c^{2} d t^{2}-d x^{2}-d y^{2}-d z^{2}
$$

Таким образом, искомое сферически-симметричное статическое решение вакуумных уравнений с $\mu=0$ представляют соотношения (12), (14) и (15). В предельном случае $\omega=\infty$ это решение переходит в соответствуюшее сферически-симметричное статическое вакуумное решение РТГ [1].

$\mathrm{C}$ целью однозначного определения $C_{1}, C_{2}$ можно ввести дополнительные условия (см., например, $[1,7]):$ а) асимптотическая псевдоевклидовость $g_{\mu \nu} ;$ б) неотрицательность $r$; в) монотонность функции $r(\zeta)$. Согласно условию “а" имеем $C_{2}=r_{1}$. При $C_{1} \neq 0$ и $\zeta \rightarrow r_{1}$ имеем или $r \rightarrow \infty$ (нарушение условия "в"), или $r \rightarrow-\infty$ (нарушение условия "б"). Таким образом, однозначно получаем решение с $C_{1}=0$ и $r=\zeta$.

Полученное решение имеет истинную сингулярность на поверхности Шварцшильда $g_{00}=0, r=r_{1}$. Рассмотрим, например, изотропный, $\gamma_{\alpha \beta} v^{\alpha} v^{\beta}=0$, вектор $E_{4}$ :

$$
v^{\alpha}=\left\{1, \frac{x}{r}, \frac{y}{r}, \frac{z}{r}\right\}
$$

Инвариант $I_{g v}=g_{\mu \nu} v^{\mu} v^{\nu}$ имеет вид

$$
I_{g v}=\left[\left(r-r_{1}\right)^{\frac{2}{k_{\omega}}}-\left(r+r_{1}\right)^{\frac{2}{k_{\omega}}}\right]\left(r+r_{1}\right)^{-k_{\omega}}\left(r-r_{1}\right)^{-\frac{(2 \omega+2)}{k_{\omega}(2 \omega+3)}}
$$

так что $I_{g v}\left(r=r_{1}\right)=-\infty, I_{g v}<0$ при $r_{1}<r<\infty$ и $I_{g v}=0$ при $r=\infty$. В соответствии с принципом причинности $I_{g v} \leqslant 0[2,3]$ гравитационное поле, которое представляет данное решение, будет физическим полем за пределами вешества только в том случае, если радиус источника поля $r>r_{1}$. Известно, что если при сжатии звездоподобного объекта выполняется граничное условие нулевого давления, то частицы на поверхности объекта движутся по времениподобным мировым линиям во внешнем вакуумном поле. Нефизичность последнего при $r \leqslant r_{1}$ означает, что частицы поверхности сжимающейся звезды не смогут пройти через барьер $r=r_{1}$, коллапс останавливается при $r>r_{1}$; в соответствии с данным решением не сушествует черных дыр, в которых происходит катастрофическое сжатие вещества до бесконечно высокой плотности.

Интегральный 4-вектор энергии-импульса замкнутой островной системы

$$
P^{\mu}=(1 / c) \int \Theta^{\mu \nu} \sqrt{-\gamma}\left(d^{3} x\right)_{\nu}
$$

в соответствии с полученным решением записывается как интеграл

$$
P^{\mu}=-\frac{k_{\omega}^{2}}{2 \varkappa c} \oint \partial_{k} \Phi^{\mu 0} n_{k} d S
$$

по сферической поверхности, лежашей в области слабого поля. Учитывая выражение $\Phi$-потенциала

$$
\Phi^{\lambda 0} \approx \frac{8 r_{0}}{r k_{\omega}^{2}} \delta_{\lambda 0},
$$


получаем корректное выражение

$$
P^{0}=M c, \quad P^{k}=0
$$

равенства “инертной” и “тяжелой” масс системы.

Рассмотрим однородную изотропную космологическую модель с плоской метрикой (15) и римановой метрикой

$$
d s^{2}=g_{\mu \nu} d x^{\mu} d x^{\nu}=\psi^{2}(t) a^{6}(t) c^{2} d t^{2}-a^{2} \delta_{m n} d x^{m} d x^{n}
$$

В пространствах (15), (17) уравнения (2) выполняются автоматически. Как обычно, материальную начинку Вселенной принимаем в виде идеальной жидкости,

$$
T_{\mu \nu}=(\varepsilon+p) u_{\mu} u_{\nu}-p g_{\mu \nu}
$$

Уравнения (5) - (7) принимают вид

$$
\begin{gathered}
\dot{\varepsilon}+\frac{3}{a} \dot{a}(\varepsilon+p)=0 ; \\
\ddot{\psi}+\frac{3}{a} \dot{a} \dot{\psi}=\frac{\varkappa}{(2 \omega+4)}(\varepsilon-3 p) ; \\
\frac{\dot{a}^{2}}{a^{2}}+\frac{\dot{a} \dot{\psi}}{a \psi}-\frac{\omega}{6 \psi^{2}} \dot{\psi}^{2}+\frac{\mu^{2}}{12}\left(\frac{1}{\psi^{2} a^{6}}+2 \psi-\frac{3}{a^{2}}\right)=\frac{\varkappa \varepsilon}{3 \psi k_{\omega}^{2}} ; \\
\frac{\dot{a} \dot{a}}{a}+\frac{\dot{a}^{2}}{a \psi}+\frac{\mu^{2}}{2}\left(\psi-\frac{1}{a^{2}}\right)=\frac{\varkappa}{(2 \omega+4) \psi}[(\omega+1) \varepsilon-\omega p],
\end{gathered}
$$

точка обозначает дифференцирование по $c \tau, d \tau=\psi a^{3} d t$. В предельном случае $\psi=$ $1, \omega=\infty$ уравнения (17), (18) - (21) переходят в соответствуюшие космологические уравнения РТГ [1]. Критерий причинности $I_{g v} \gtrless 0$ при $v^{\alpha}(16)$ принимает вид

$$
\psi^{2} a^{4} \leqslant 1
$$

т.е. при эволюции Вселенной величина $\psi^{2} a^{4}$ не может превысить определенного максимального значения. Интересно отметить, что соотношение (22) само по себе совместимо как с циклическими несингулярными моделями, так и с моделями “вечной” Вселенной с неограниченным ростом масштабного фактора и одновременным уменьшением гравитационного скаляра. Конкретно отметим приближенное решение для начала Вселенной с регулярным минимумом масштабного фактора

$$
a(\tau=0)=a_{\min } \equiv a_{0}>0, \quad \dot{a}(\tau=0)=0, \quad \ddot{a}(\tau=0)>0
$$

и регулярным максимумом скалярного поля

$$
\psi(\tau=0)=\psi_{\max } \equiv \psi_{0}>0, \quad \dot{\psi}(\tau=0)=0, \quad \ddot{\psi}(\tau=0)<0
$$


При $\tau \sim 0$ это решение имеет вид

$$
\begin{aligned}
& a \approx a_{0}+\frac{c^{2} \tau^{2}}{a_{0} \psi_{0}(4 \omega+8)}\left\{\varkappa a_{0}^{2}\left[(\omega+1) \varepsilon_{0}-\omega p_{0}\right]+\mu^{2} \psi_{0}(\omega+2)\left(1-\psi_{0} a_{0}^{2}\right)\right\}, \\
& \psi \approx \psi_{0}+\frac{\varkappa\left(\varepsilon_{0}-3 p_{0}\right)}{(4 \omega+8)} c^{2} \tau^{2},
\end{aligned}
$$

где

$$
\begin{aligned}
& \varepsilon_{0} \equiv \varepsilon(\tau=0)=\varepsilon_{\max }, \quad p_{0} \equiv p(\tau=0), \\
& \varepsilon_{0}=\left(\mu^{2} k_{\omega}^{2} / 4 \varkappa \psi_{0} a_{0}^{6}\right)\left(1+2 \psi_{0}^{3} a_{0}^{6}-3 \psi_{0}^{2} a_{0}^{4}\right), \\
& \varkappa\left[(\omega+1) \varepsilon_{0}-\omega p_{0}\right]+\mu^{2} \psi_{0}(\omega+2)\left(\frac{1}{a_{0}^{2}}-\psi_{0}\right)>0, \\
& \varepsilon_{0}-3 p_{0}<0 .
\end{aligned}
$$

Уравнение состояния будем предполагать в виде (см., например, [1])

$$
p=\nu \varepsilon, \quad 0 \leqslant \nu \leqslant 1 .
$$

В соответствии с нашим решением имеем условия

$$
\begin{gathered}
1+2 \psi_{0}^{3} a_{0}^{6}-3 \psi_{0}^{2} a_{0}^{4}>0 \\
1+\omega(1-\nu)+\psi_{0}^{2} a_{0}^{4}\{3+\omega(1+3 \nu)\}-2 \psi_{0}^{3} a_{0}^{6}\{2+\omega(1+\nu)\}>0 \\
\nu>1 / 3 .
\end{gathered}
$$

Комбинируя (24) и (25), получаем важное неравенство

$$
\psi_{0}^{2} a_{0}^{4}<1
$$

которое соответствует условию причинности (22).

Автор признателен профессору Г. В. Шишкину за внимание к работе и полезное обсуждение.

\section{Список литературы}

[1] Логунов А.А., Мествиришвили М.А. Релятивистская теория гравитации. М.: Наука, 1989.

[2] Логунов А. А. // ТМФ. 1992. Т. 92. №2. С. 191.

[3] Логунов А. А. // ТМФ. 1994. Т. 101. №1. С. 3.

[4] Brans C., Dicke R. H. // Phys. Rev. 1961. V. 124. № 1. P. 925.

[5] Noerdlinger P. D. // Phys. Rev. 1968. V. 170. №5. P. 1175.

[6] Уилл K. Теория и эксперимент в гравитационной физике. М.: Энергоатомиздат, 1985.

[7] Бронников К. А., Шикин Г. Н. // ТМФ. 1988. Т. 76. №2. С. 304.

Белорусский государственный

Поступила в редакцию педагогический университет

5.V.1995 г.

\section{V.N. Tuniak}

\section{RELATIVISTIC THEORY OF GRAVITY AND BRANS-DICKE SCALAR FIELD}

Relativistic theory of gravity (RTG) is generalized by including the Brans-Dicke-type scalar field in the Minkowski space. The corresponding scalar-tensor theory is discussed in detail. 